\title{
Horizontal mixing in shallow flows
}

\author{
W.S.J. Uijttewaal ${ }^{1}$ \\ ${ }^{1}$ Environmental Fluid Mechanics Section \\ Delft University of Technology \\ THE NETHERLANDS \\ E-mail: w.s.j.uijttewaal@tudelft.nl
}

\begin{abstract}
Shallow shear flows play an important role in the transverse transport of mass and momentum in rivers. In order to understand structure of the flow and the mechanism resulting in the particular turbulence properties, three types of shallow mixing layers are investigated each with a different cause for the velocity difference: inflow conditions, bed level and bed roughness. It is shown that mixing layer properties are highly affected by the 3D turbulence generated in the bottom boundary layer. This implies that effects of subtle geometric properties like roughness variation and bed level changes should be incorporated in predictive models
\end{abstract}

Keywords: shallow flow, mixing, secondary circulation, compound channel, roughness

\section{INTRODUCTION}

Predicting the flow in rivers accurately is important for flood control, river engineering and measures that are taken for safeguarding or increasing the discharge for a given maximum water level. As rivers are rather variable regarding discharge, as well as bathymetry the flow is continuously adapting to its boundary conditions. The associated velocity gradients lead to the production of large eddies that can contribute substantially to transverse exchange of mass and momentum. It is therefore important to understand the generation mechanisms of coherent structures as well as their development. As current computational resources do not allow solving for the flow in river reaches with a resolution high enough to resolve the large eddy structures, these phenomena have to be accounted for in a simplified and parameterised form. A proper understanding of the phenomena helps in formulating these parameterisations.

Many environmental flows such as rivers are bounded in the vertical by a free surface and a bed. The vertical confinement puts constraints to the length scale by which momentum is exchanged between the bed and the water column. The properties of the bed are therefore of paramount importance for the overall flow pattern. Due to the space available, mixing lengths in the horizontal directions can however be much bigger thus allowing for more effective mixing and momentum exchange. For these large mixing lengths to develop in the presence of high dissipation the shear layer should be hydrodynamically unstable giving rise to the accumulation of kinetic energy in large eddy structures. The shallowness causes the motion of these structures to lie predominantly in the horizontal plane resulting in quasi two-dimensional features.

This paper addresses the various mechanisms that govern the horizontal exchange of mass and momentum in shallow mixing layers. From the classical examples of mixing layers, wakes and jets the first one has our main interest. This is mainly because the mixing layer is found in many applications like river confluences, compound channels, groyne fields and harbour entrances. It is also the archetype of a simple unstable shear flow which is well studied for a large variety of conditions.

\section{SHALLOW MIXING LAYERS}

The shallow mixing layers in this paper are defined as open channel flows with a transverse velocity gradient. It is shallow because the mixing layer width is generally larger than the water depth. The lateral velocity difference giving rise to the shear layer in the mean flow can have various causes depending on the specific boundary conditions: 


\subsection{Differences in inflow velocities as it is found with river confluences, lateral expansions and side cavities}

Two regions with different streamwise velocities form a mixing layer at the interface between them (Uijttewaal \& Booij 2000). With a uniform horizontal bed and a transversely uniform free-surface slope the velocity difference is disappearing gradually with downstream distance because the high-velocity side is decelerated by bed friction whereas the low velocity side is accelerated (Talstra et al. 2006). The eventual transversely uniform flow is established through friction and gravity rather than through a horizontal momentum exchange. Nevertheless, the instabilities in the shear layer develop into eddy structures that give rise to an increase in the horizontal mixing length and consequently a growth of the mixing layer width. The figure shows that the mixing layer width can grow to more than 10 times the water depth over a distance of approximately 100 times the water depth.

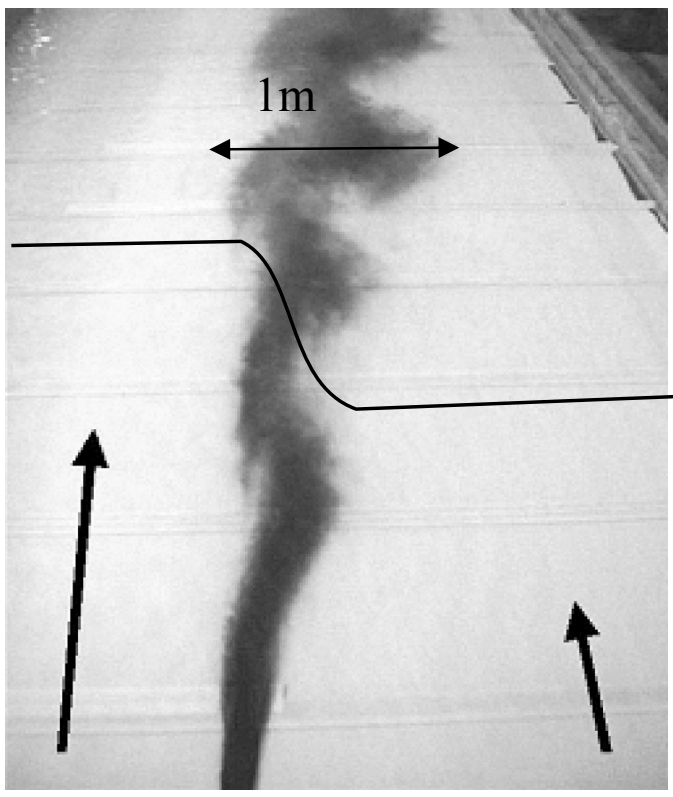

Figure 1: Perspective view on the visualisation of a shallow mixing layer in a horizontal flat-bed laboratory flume, $3 \mathrm{~m}$ wide, $67 \mathrm{~mm}$ deep, showing large eddies up to $1 \mathrm{~m}$ diameter as well as the small-scale diffusive bottom turbulence (Van Prooijen \& Uijttewaal 2002). The arrows indicate the mean streamwise velocities of $0.3 \mathrm{~m} / \mathrm{s}$ and $0.1 \mathrm{~m} / \mathrm{s}$, determined by inflow conditions.

\subsection{Lateral variation in water depth as it is found with compound channels with shallow floodplains and a deep main channel.}

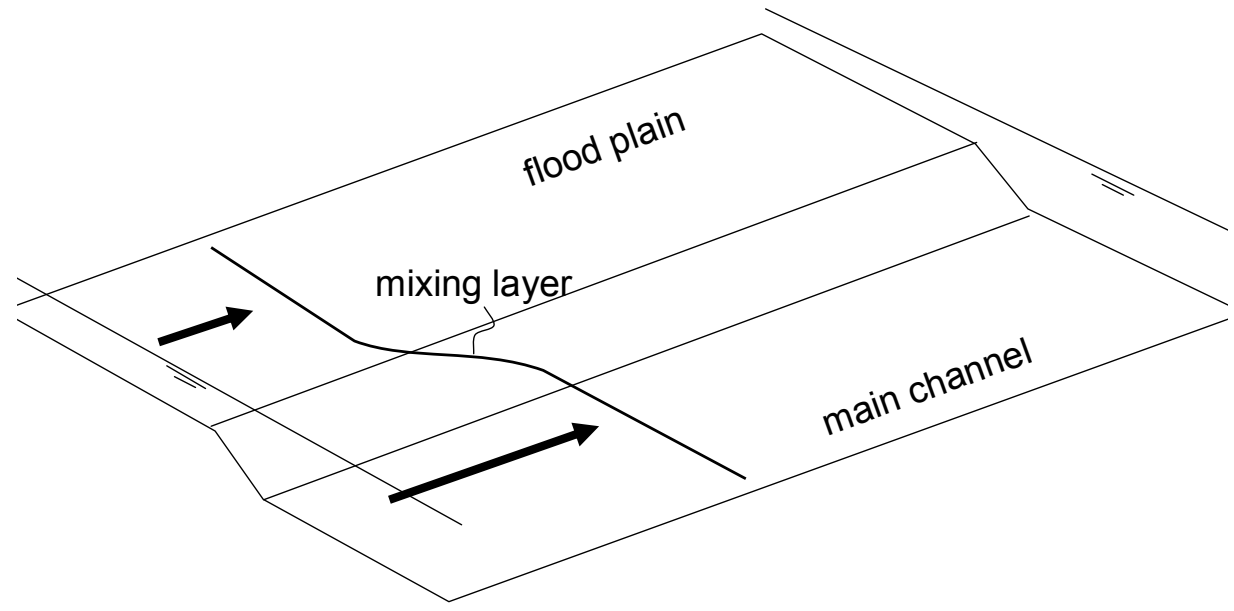

Figure 2: Sketch of a mixing layer generated by a transverse difference in water depth 
A schematised version of a river with a high water stage, a so called compound channel flow, is depicted in figure 2. In the main channel and the flood plain two parallel streams are formed with different velocities in accordance with the depth. The flow in the shallower part experiences higher friction resulting in a lower mean velocity. The interfacial shear layer leads to the formation of eddy structures that contribute to the momentum transfer from the main channel to the floodplain. Although this mixing layer looks very similar to the previous configuration the effect of the transverse depth variation is that the transverse velocity difference will not disappear with downstream distance. Furthermore, any transverse motion in the mixing layer will sense the variation in depth (Van Prooijen et al. 2005).

\subsection{Lateral variation in bed friction due to variation in bed material, bed forms or vegetation}

In natural systems the bed is seldom smooth and roughness distributions can be heterogeneous on various scales. In order to study what happens at the transitions from hydraulically smooth to rough beds in streamwise and transverse directions a geometry was arranged as depicted in figure 3 , since in comparison with a smooth bed the flow above a rough bed attains a lower mean velocity. Above the transition between the smooth and the rough bed a mixing layer develops. As with the compound channel case, at a certain downstream distance an equilibrium situation establishes for the transverse distribution of the streamwise velocity. For the case shown it is of the order of 50 times the depth. In contrast with the mixing layer of figure 1 , the width of the mixing layer remains of the order of the water depth indicating that another mechanism is governing the mixing (Vermaas et al. 2011).
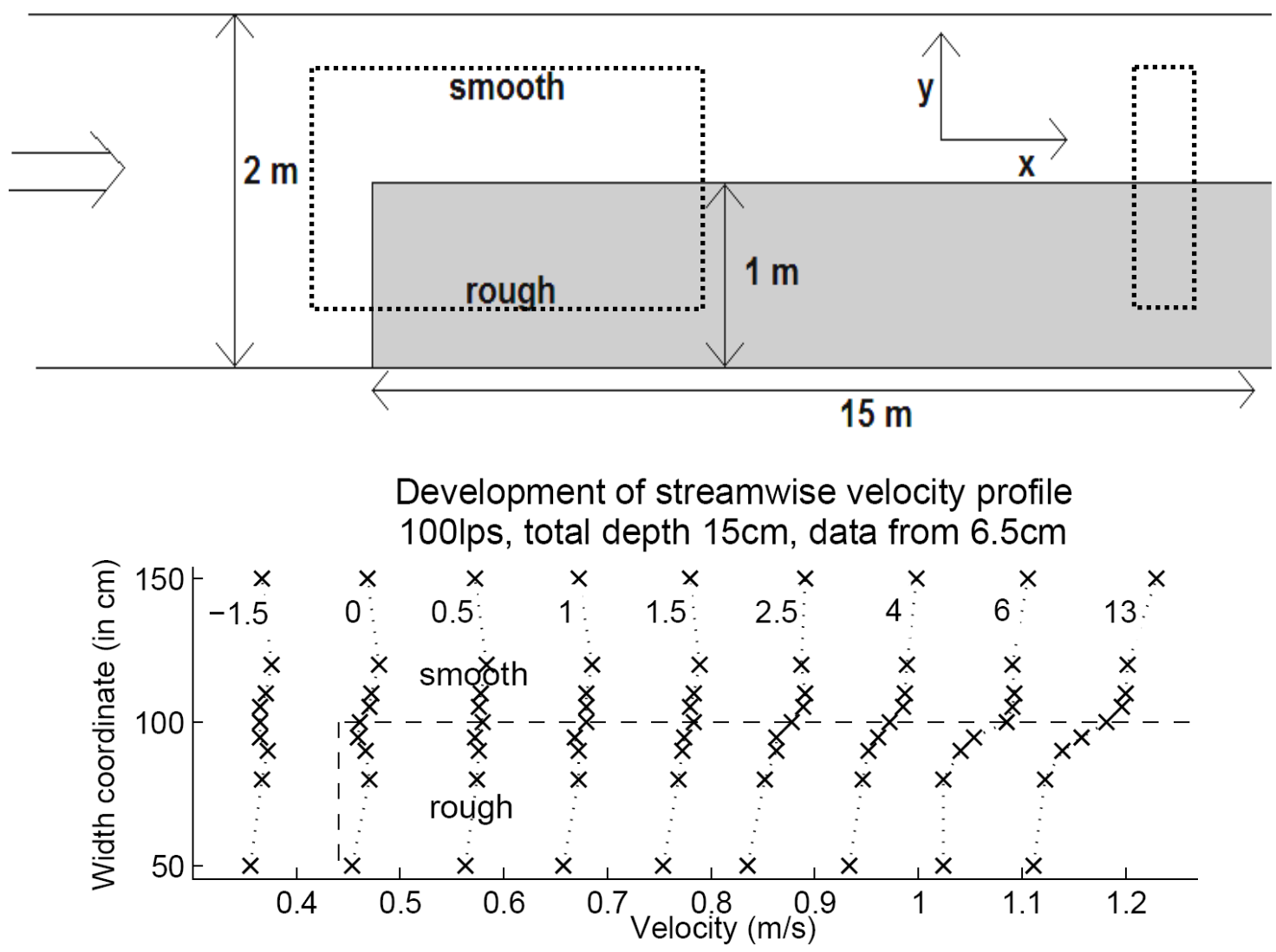

Figure 3: Sketch of an experiment on mixing layer formation due to roughness variation. Top view of the experimental configuration (upper panel). Measured transverse profiles of streamwise velocity are labelled with downstream distance $(\mathrm{m})$ and the velocity scale is shifted $0.1 \mathrm{~m} / \mathrm{s}$ for each curve (lower panel) (Vermaas et al. 2011). 
The three examples as addressed here all lead to the formation of a shallow shear layer. The development of the shear layer and the coherent structures therein will be different for each case, despite the fact that the profiles of mean velocity can look very similar.

Another complicating factor is that in natural systems often a combination of the three causes is found. For example, the shallow flood plains are usually covered with vegetation whereas the deeper main channel is not. Moreover, with movable beds the roughness in the form of ripples and dunes can develop in mutual interaction with the flow thereby affecting the flow resistance.

\section{FLOW STUCTURES}

The turbulence in the shear layer is characterised by three types of flow structures: large-scale quasi$2 \mathrm{D}$ eddies with vertical vorticity, small-scale 3D turbulence, and secondary circulation with streamwise vorticity. The dominance of one over the other with respect to the transfer of momentum will depend on the specific flow configuration and the turbulence generation mechanism.

\subsection{Quasi-2D turbulence}

Considering the shallow mixing layer of the geometry of figure 1 from a depth averaged perspective, the shear induced Kelvin-Helmholz instabilities result in vortex structures with vertical axes of rotation. From a linear stability analysis that includes bed shear stress and the effective eddy viscosity due to small scale 3D turbulence, a range of wave lengths can be identified that have a positive growth rate. A structure that is advected downstream will grow in accordance with its size and the local mean velocity profile. The energy density distribution for a certain downstream position can therefore be obtained by calculating the accumulated growth along the mixing layer for each wave length. An example of such an analysis is given in figure 4 together with experimental data (Van Prooijen \& Uijttewaal 2002). It shows that the spectral distribution for the large scale motion is reasonably well predicted and that the energy level from which the structures start to grow is that of the background turbulence (indicated by the horizontal line). The latter conclusion indicates the importance of a correct representation of the disturbances that form the seedlings of the eddy structures. It was demonstrated by Van Prooijen and Uijttewaal (2008), that for a depth averaged simulation of a mixing layer the technique of kinematic simulation is an effective tool.
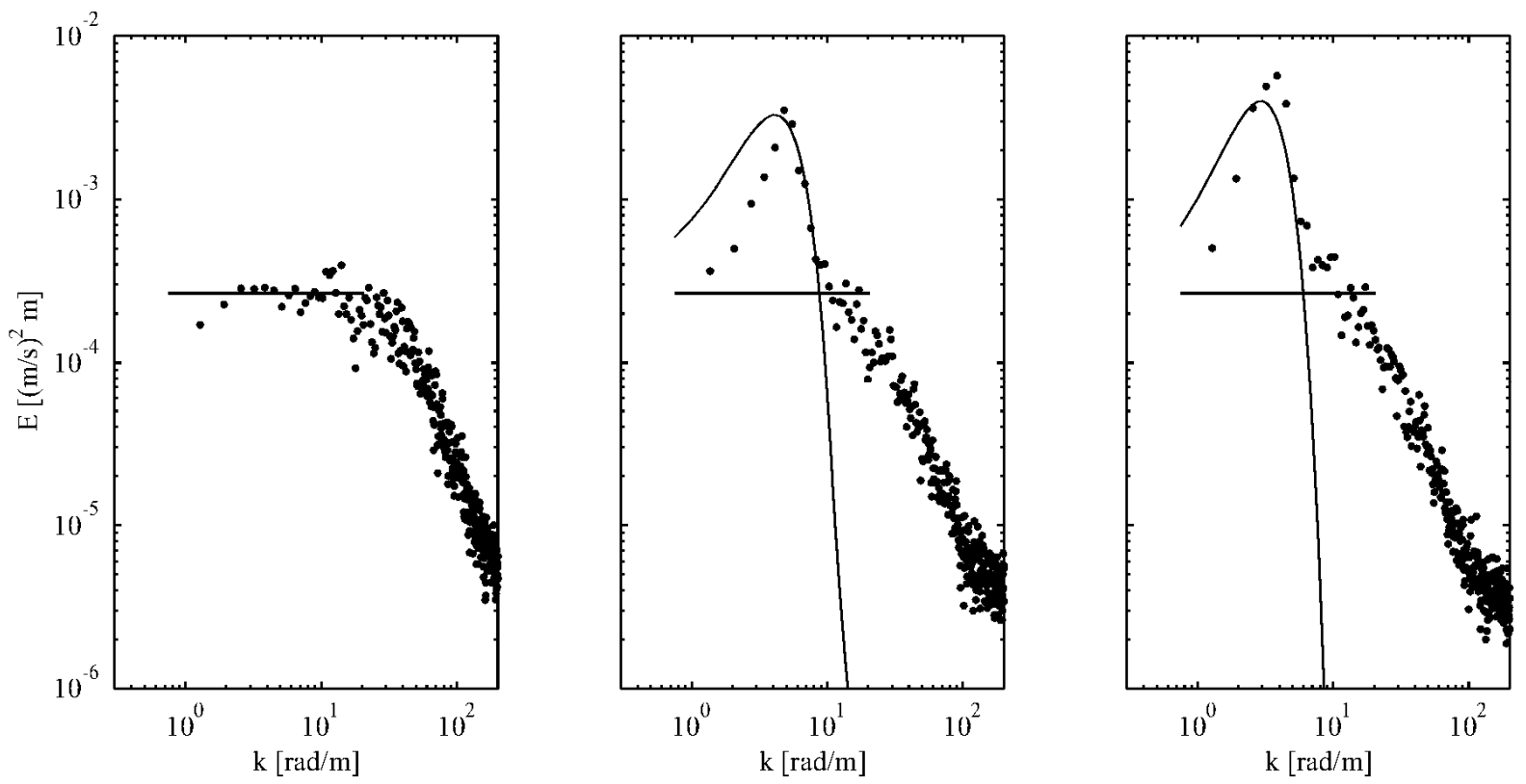

Figure 4: Energy density spectra of a shallow mixing layer (depth=7 $\mathrm{cm}$ ) obtained at $0,4.5$ and $10 \mathrm{~m}$ downstream of the splitter plate. Accumulated growth rates of the stability analysis of the large scales are compared with experimental data. 
For this example of a transversely uniform geometry the momentum exchange, and thus the mixing, is dominated by the quasi-two-dimensional large-scale eddies where the role of bed friction and turbulence can be modelled in a simple and straightforward way.

\subsection{Effects of transverse depth variation}

As the mixing layer is found above the transition from the main channel to the flood plane any transverse motion in that area will be affected by the change in depth (Fig. 5). The vertical compression will accelerate the flow towards the flood plain and decelerate the reverse flow leading to a deformation of the eddy structures. This effect is supposed to enhance mixing proportional to the relative change in water depth (Van Prooijen et al. 2005).

Implementing this idea in a simple momentum balance produces good agreement with experimental data. Figure 6 shows that the mixing layer shape and stress distribution is well captured by the model. It should be noted that the good agreement is only an indirect justification of the model assumptions. There are no direct observations of the eddy structures in those experiments.

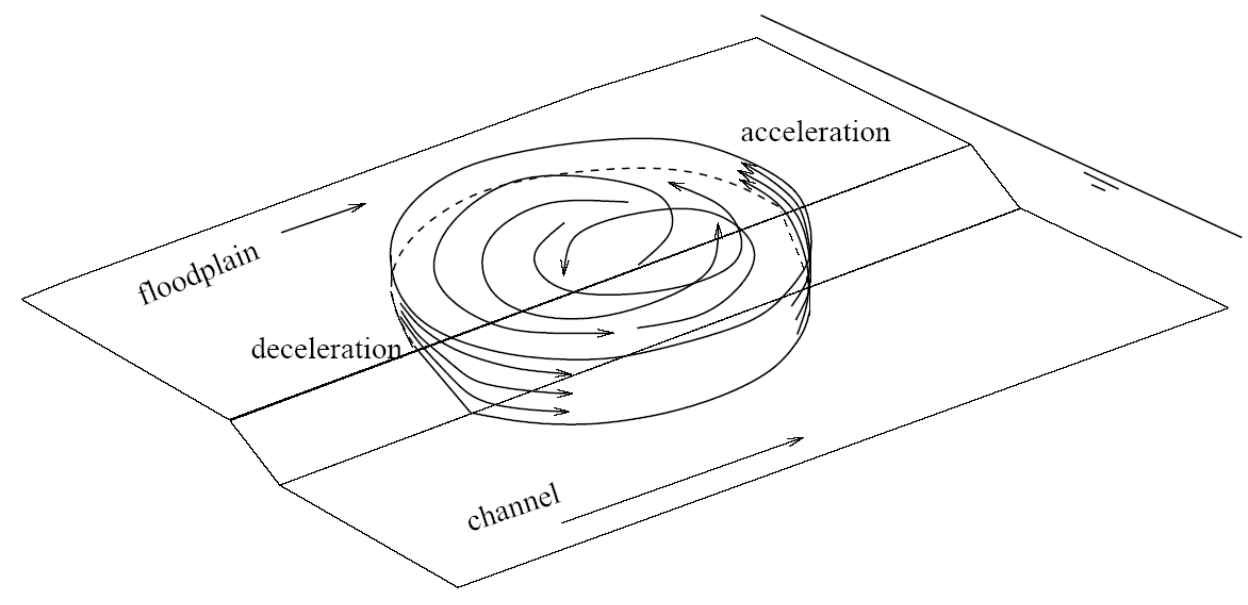

Figure 5: A large eddy moving along the interface of a lateral depth variation.
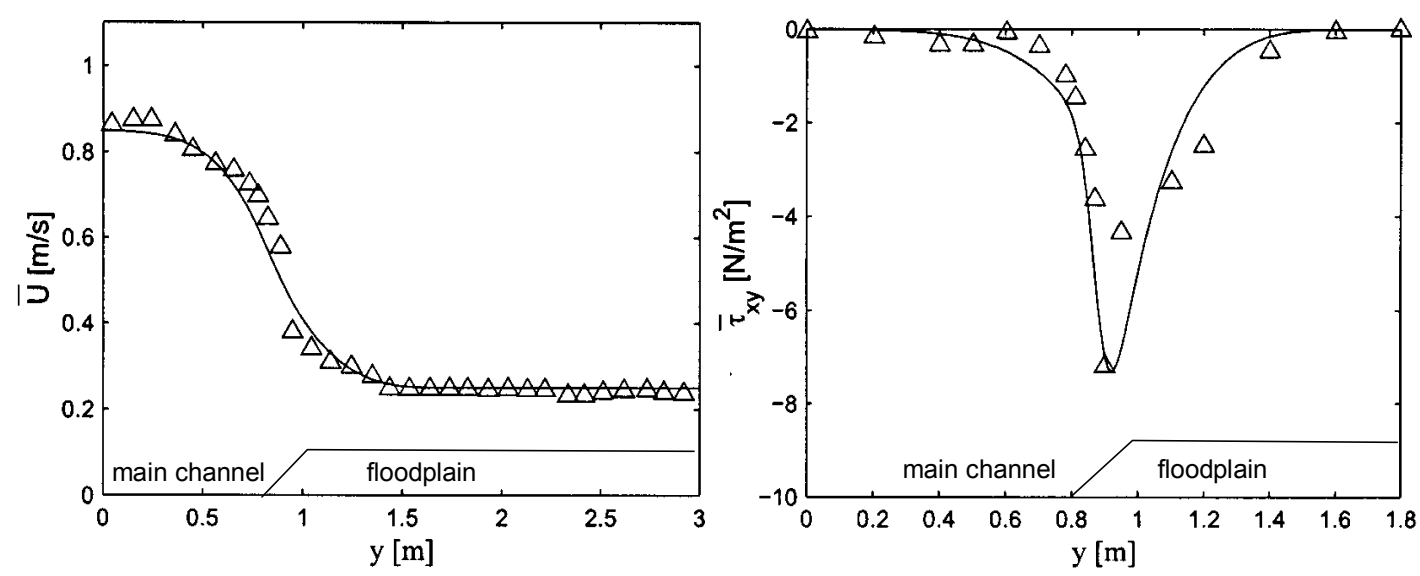

Figure 6: Comparison of data from compound channel experiments with depth ratio $D_{\mathrm{fp}} / D_{\mathrm{mc}}=0.11$ (Knight \& Shiono 1990), with a model that accounts for transvers depth variation (Van Prooijen et al. 2002). Transverse profile of mean streamwise velocity (left). Profile of interfacial shear stress (right). 


\subsection{Heterogeneous roughness}

With the two previous cases in mind one would think to find similar observations for the mixing layer caused by roughness variation. Surprisingly, no large eddy structures are found in this case. This explains why the mixing layer in figure 3 remains narrow despite the large downstream distance. Apparently the water depth determines the dominant length scale of mixing. For this configuration the transverse roughness change gives rise to a circulation cell in the plane perpendicular to the main stream. Though the magnitude of the transverse velocity is small, it is large enough to prevent mixing layer eddies to be formed.

In figure 7 experimental data are compared with a Large Eddy Simulation of the flow. As the transverse velocities have a magnitude of only a few percent of the streamwise velocities, the experimental data are a bit noisy. Furthermore the acoustical device did not allow to measure velocities in the upper $5 \mathrm{~cm}$ of the water column. It is clear from figure 7 that near the bed at the transition the flow is pushed away from the rough towards the smooth side. The formation of streamwise vorticity is known to occur where the anisotropy in the turbulence is strong (Perkins 1970). The corner eddies in a straight open channel are examples of the same phenomenon. The strength of the cell is influenced by the abruptness of the lateral change in bed roughness. The large eddy simulation results were obtained on a domain with streamwise periodic boundary conditions and a stress-free rigid lid surface whereas the roughness was imposed in the bottom boundary conditions. As the circulation cell is bounded by the vertical dimensions of the flow the mixing layer width is also restricted to this size. Any large-scale structure that would develop in the mixing layer is advected and deformed by the circulation cell before it can attain a significant amplitude.
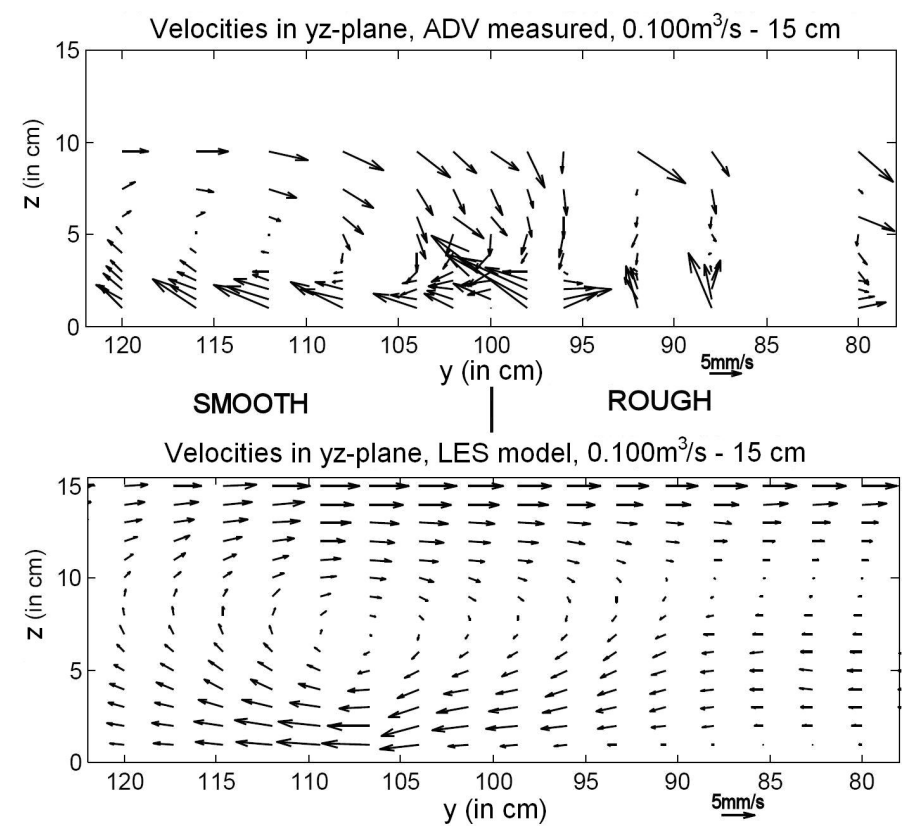

Figure 7: Time averaged velocities in a cross-sectional plane in a developed flow over parallel lanes that differ in roughness. Top panel: ADV measurements. Lower panel: LES computations (with data interpolated to a coarse grid).

It is important to notice that for all cases mentioned, boundary layer turbulence is present with an essentially 3D nature and a length scale typically smaller than the water depth. This 'background' 3Dturbulence is found throughout the whole flow domain and is not restricted to the mixing layer. However it has an effect on the generation of eddy structures because it acts as a disturbance on the main flow. At the same time its dissipative character drains energy from the large eddies.

\subsection{Secondary circulation}

The secondary circulation in a straight flow is generally weak, as the driving force stems from second order effects in the turbulent stresses and is generally labeled as secondary flow of the second kind. In 
the practice of river engineering however the flow geometry is usually curved giving rise to a secondary flow and resulting in a significant transport across the flow. This type of secondary flow is naturally governed by the flow curvature and is labeled as secondary flow of the first kind and only to a minor extent affected by the anisotropy of the occurring turbulent stresses. This transverse transport of momentum results, for sharp bends, in area's of low streamwise velocity near the inner bank and of relatively high velocities near the outer bank. Such a redistribution of momentum thus contributes to the mixing over the cross-section as well as to the dispersive properties of the flow.

\section{CONCLUSIONS}

The examples of shallow mixing layers provided in this paper reveal that they can be formed under various conditions. Despite the fact that the mean streamwise velocity distribution is simple and can generally be considered as two-dimensional (i.e. uniform over the depth), subtle 3D features affect the horizontal mixing substantially. These are mainly related to the properties of the bed and the smallscale turbulence generated in the boundary layer. In order to properly predict the horizontal mixing the effects of bottom boundary layer should be represented in the modelling approach. This requires either a (large eddy) simulation with a resolution sufficiently high so that the energy containing part of the 3D turbulence spectrum is resolved, or a proper parameterisation of its effects on the large-scale flow. The former has become feasible for schematised laboratory experiments at moderate Reynolds numbers. The latter will still be necessary during the coming decades when it concerns the simulation of high Reynolds-number river flows on a prototype scale.

\section{ACKNOWLEDGMENTS}

This work was conducted in collaboration with Bram van Prooijen, Wim van Balen, Harmen Talstra, David Vermaas en Ton Hoitink.

\section{REFERENCES}

Balen W. van, Uijttewaal W.S.J. and Blanckaert K.J.F., (2010) Scalar dispersion in strongly curved open-channel flows. In Proceedings of the International conference on Fluvial Hydraulics pp. 169-177.

Knight D. W. and Shiono K., (1990) Turbulence measurements in a shear layer region of a compound channel. J. Hydraul. Res., 28(2), 175-196.

Perkins H.J. (1970) The formation of streamwise vorticity in turbulent flow. J. Fluid Mech., 44(4), 721740.

Prooijen B.C. van, and Uijttewaal W.S.J., (2002) A linearized model for the evolution of large-scale turbulence structures in shallow mixing layers Physics of Fluids, 14 (12), 4105-4114.

Prooijen B.C. van, Battjes J.A. and Uijttewaal W.S.J., (2005) Momentum exchange in uniform compound channel flow. J. Hydraulic Engineering Vol 131(3) pp. 175-183.

Prooijen B.C. van and Uijttewaal W.S.J. (2009) The relevance of a back-scatter model for depthAveraged flow simulation, Flow Turbulence and Combustion, 82(1), 73-91.

Talstra H, Uijttewaal W.S.J. and Stelling, G.S.,(2006) Emergence of large-scale coherent structures in a shallow separating flow. In RML Ferreira \& ECTL Alves (Eds.), River flow 2006 pp. 261-269 London: Taylor\&Francis.

Uijttewaal W.S.J. and Booij R., (2000) Effects of shallowness on the development of free-surface mixing layers. Physics of Fluids 12(2) 392-402.

Vermaas, D. A., W. S.J. Uijttewaal, and A.J.F. Hoitink (2011), Lateral transfer of streamwise momentum caused by a roughness transition across a shallow channel, Water Resour. Res., 47, W02530, doi:10.1029/2010WR010138. 